Document downloaded from:

http://hdl.handle.net/10251/36653

This paper must be cited as:

Chen Charpentier, BM.; Cortés López, JC.; Romero Bauset, JV.; Roselló Ferragud, MD. (2013). Some recommendations for applying gPC (generalized polynomial chaos) to modeling: An analysis through the Airy random differential equation. Applied Mathematics and Computation. 219(9):4208-4218. doi:10.1016/j.amc.2012.11.007.

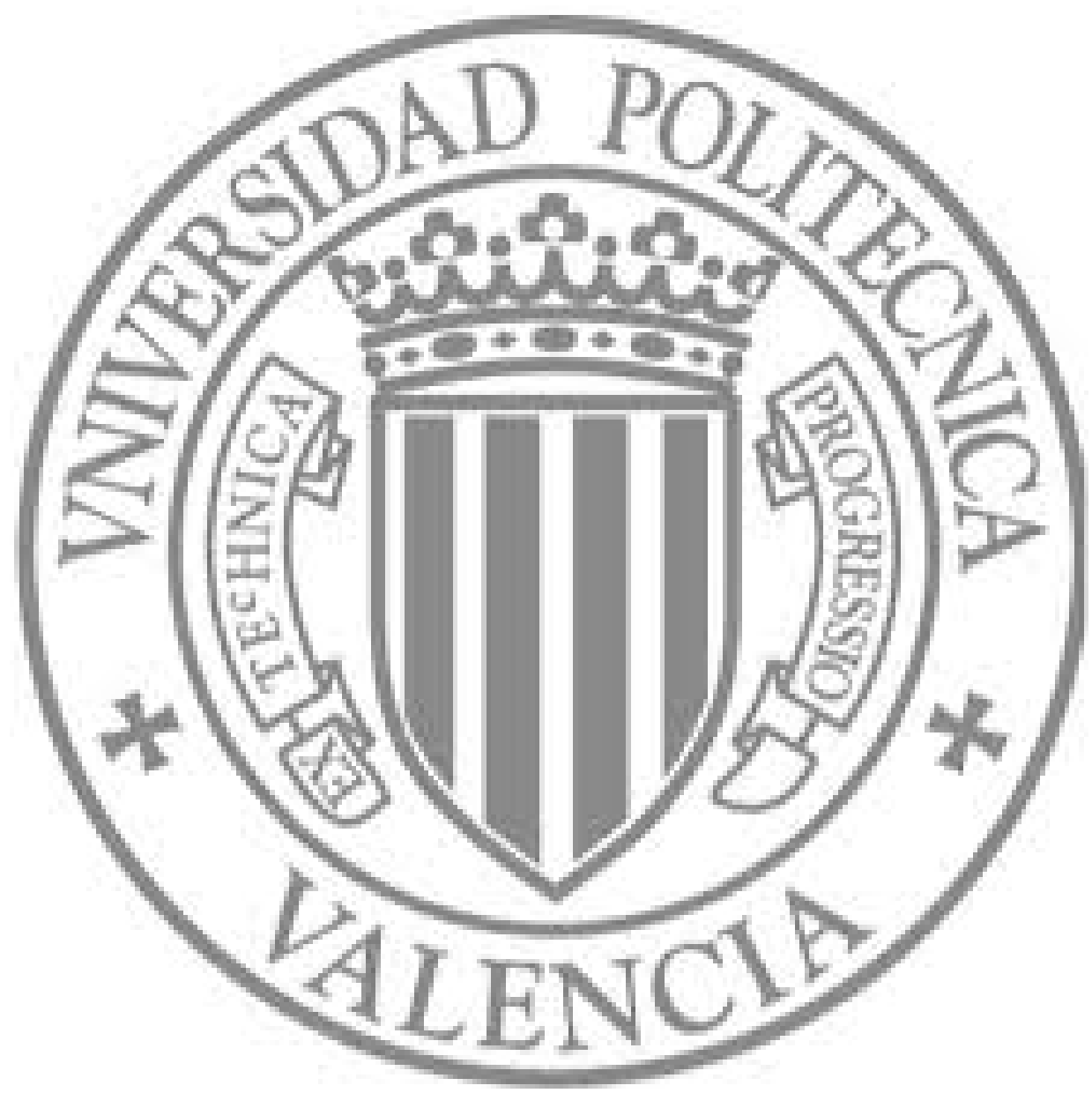

The final publication is available at

http://dx.doi.org/10.1016/j.amc.2012.11.007

Copyright Elsevier 


\title{
Some recommendations for applying gPC (generalized Polynomial Chaos) to modelling: An analysis through the Airy random differential equation
}

\author{
Benito M. Chen-Charpentier ${ }^{\mathrm{a}}$, J.-C. Cortés ${ }^{\mathrm{b}}$, J.-V. Romero ${ }^{\mathrm{b}, *}$, M.-D. \\ Rosellób \\ ${ }^{a}$ Department of Mathematics, University of Texas at Arlington, Arlington, TX \\ 76019-0408, United States \\ ${ }^{b}$ Instituto Universitario de Matemática Multidisciplinar, Universitat Politècnica de \\ València, Camino de Vera s/n, 46022, Valencia, Spain
}

\begin{abstract}
In this paper we study the use of the generalized polynomial chaos method when the differential equations describing the model depend on more than one random input, whether parameters or initial or boundary conditions. We study the effect of the choice of density distribution functions of the inputs on the output stochastic processes. Specifically we study the effect on the solutions of Airy's equation which is good test case since the solutions are highly oscillatory and errors develop both in the amplitude and the phase. Several different cases are considered and conclusions are presented.
\end{abstract}

Keywords: Airy random differential equation, generalized Polynomial Chaos (gPC)

1. Introduction and motivation

Traditionally mathematical models based on deterministic differential 3 equations have been considered to describe numerous phenomena appearing 4 in scientific areas such as engineering, physics, medicine, economics or biol-

\footnotetext{
*Corresponding author

Email addresses: bmchen@uta.edu (Benito M. Chen-Charpentier), jccortes@imm.upv.es (J.-C. Cortés), jvromero@imm.upv.es (J.-V. Romero), drosello@imm.upv.es (M.-D. Roselló)
}

October 29, 2012 
5

ogy. There is a great deal of experience in the use of such models, but their application requires accurate knowledge of the data of the model, namely, the input coefficients and the initial/boundary conditions given either by constants and/or deterministic functions. Often the data can only be established roughly since it may depend on experimental measurements. Also the consideration not only of errors in the observed or measured data, but also the variability and uncertainty inherent to the complexity of the phenomenon under study, leads to consider that both, input coefficients and initial/boundary conditions, are random variables (r.v.'s) and/or stochastic processes (s.p.'s) rather than deterministic quantities. These facts motivate the need to consider random differential equations (r.d.e.'s) to describe the behavior of quantities of interest instead of their deterministic counterparts. As a consequence, numerous mathematical models based on r.d.e.'s have been proposed over the last few decades in a wide variety of applied areas $[1,2,3,4,5,6]$.

In practice, once the differential equation model has been selected, the determination of the statistical distribution for each random input and initial/boundary condition is required. Afterward, one deals with the computation of the solution s.p. including its main statistical functions such as average and standard deviation (or equivalently, variance). To tackle this task a considerable number of methods have been developed $[7,8,9,10,11$, $12,13,14,15,16,17]$. Here we are specifically interested in generalized polynomial chaos (gPC) technique $[18,19]$ that has been shown to be relatively easy to implement and to give good results for several application models.

$\mathrm{gPC}$ is a powerful method to represent, by means of infinite series, secondorder r.v.'s. These series are defined in terms of the Wiener-Askey scheme which uses common discrete and continuous orthogonal polynomials as basis functions to represent the random inputs and outputs solutions of the model equations. Taking into account that some of the weighting functions associated to these orthogonal polynomials are identical to the probability function of certain statistical distributions including the standard families such as Binomial, Negative Binomial, Hypergeometric, Poisson, Gaussian, Beta, Gamma, gPC allows the variables to be expanded with respect to suitable orthogonal polynomial bases, that in the previous list correspond to Krawtchouk, Meixner, Hahn, Charlier, Hermite, Jacobi, Laguerre, respectively [19]. gPC takes advantage of this key result in dealing with one of its most fruitful applications: the solution of r.d.e.'s. In fact, in the outstanding paper [19], authors show, through the exponential population growth model 
$\dot{Y}(t)=-K Y(t), Y(0)=1$ (whose decay rate coefficient $K$ is assumed to be a r.v. following different standard statistical distributions) that, an exponential convergence of the error measures for the average and variance of the solution takes place when the series representation of the solution s.p. is made in terms of (an optimum) trial polynomial basis from the Wiener-Askey scheme in accordance with the distribution of random input $K$.

In the usual case where there are more than one random input parameter, each having possibly different probability distributions, gPC can still be employed. This is usually done by using a single orthogonal polynomial basis, although there is no criterion to choose the best basis. Frequently one opts to represent the solution s.p. as well as the random model parameters using the Hermite orthogonal polynomial basis which is linked with Gaussian r.v.'s $[18,20]$. Likely this decision can initially be motivated by the well-known role that Gaussian r.v.'s play in Probability Theory to represent asymptotically many relevant r.v.'s according to the Central Limit Theorem. However in dealing with random differential models this decision may not be adequate since each random parameter plays a different role in the model, such as, diffusion coefficient, source term, initial condition, boundary condition, etc. As a consequence, they contribute differently in determining the behavior of the solution.

Assuming different statistical distributions for each of the random model parameters and, bearing in mind the idea of developing them with respect to one single gPC basis, in this paper we first explore the advisability of representing the solution s.p. in other bases likely different from Hermite polynomials. Following the gPC method, this trial orthogonal basis is set in accordance with the statistical distribution of the random parameter that most contributes to determine the behavior of the model. Second, in order to improve the results provided by previous approach, we also analyze the possibility of computing the solution s.p. when the random model parameters are represented in different bases. To conduct our study, we have chosen the Airy r.d.e.

$$
\ddot{X}(t)+A t X(t)=0, \quad t>0, \quad X(0)=Y_{0}, \quad \dot{X}(0)=Y_{1},
$$

because it is well-known that the solution of the deterministic Airy differential equation is highly oscillatory, hence it is expected that, in dealing with its stochastic counterpart, differences, if any, between the solutions obtained by gPC using different orthogonal polynomial bases will be highlighted. 
This paper is organized as follows. In Section 2, we summarize the gPC method focusing on its application to solve the r.d.e. (1). Section 3 is devoted to show the numerical results obtained to the study previously described. Conclusions and suggestions are drawn in Section 4.

\section{Applying gPC to the random Airy differential equation}

This section is concerned with introducing the gPC method including its application in the construction of approximate solution s.p. to problem (1). Henceforth we shall assume that coefficient $A$ and initial conditions $Y_{0}$ and $Y_{1}$ are independent r.v.'s defined on a common probability space $(\Omega, \mathcal{F}, P)[21$, part I]. Thus, r.v.'s $A, Y_{0}$ and $Y_{1}$ depend on an outcome $\omega \in \Omega$, i.e., $A=A(\omega)$, $Y_{0}=Y_{0}(\omega), Y_{1}=Y_{1}(\omega)$. As a consequence, the solution $X(t)=X(t ; \omega)$ to problem (1) is a s.p.

The polynomial chaos method was firstly introduced by N. Wiener who called it the homogeneous chaos [22]. He used expansions in Hermite polynomials. In 2002, Xiu et al. [19] introduced the generalized polynomial chaos, which allows to use the polynomials of the Wiener-Askey scheme. In this context, if $L_{2}$ denotes the set of all r.v.'s $\chi$ whose statistical second-order moments with respect to the origin are finite, i.e., r.v.'s such that $\left\langle\chi^{2}\right\rangle<+\infty$, (where $\langle\cdot\rangle$ denotes the expectation operator), and, as a consequence, also has finite variance, then every $\chi \in L_{2}$ can be represented in the form

$$
\chi(\omega)=\widehat{\chi}_{0} \Gamma_{0}+\sum_{i_{1}=1}^{\infty} \widehat{\chi}_{i_{1}} \Gamma_{1}\left(\xi_{i_{1}}(\omega)\right)+\sum_{i_{1}=1}^{\infty} \sum_{i_{2}=1}^{i_{1}} \widehat{\chi}_{i_{1} i_{2}} \Gamma_{2}\left(\xi_{i_{1}}(\omega), \xi_{i_{2}}(\omega)\right)+\cdots
$$

where $\Gamma_{i}$ are successive Wiener-Askey polynomial chaoses which depend on $i$ independent r.v.'s of vector $\boldsymbol{\xi}=\left(\xi_{i_{1}}, \xi_{i_{2}}, \ldots\right)$. These polynomials $\Gamma_{i}$ have increasing degrees starting from zero $[22,18,19]$. It has been demonstrated that this expansion converges, in the particular case of Hermite polynomials, for second-order s.p.'s [23]. As a consequence, the two first terms in the representation (2) can be interpreted as the Gaussian part of r.v. $\chi$.

For convenience, this representation can be arranged using a given polynomials basis $\mathcal{B}=\left\{\Phi_{j}\right\}$ as

$$
\chi(\omega)=\sum_{j=0}^{\infty} \chi_{j} \Phi_{j}(\boldsymbol{\xi}(\omega))
$$


since there is a one-to-one correspondence between $\Phi_{j}(\cdot)$ and $\Gamma_{i}(\cdot) . \quad\left\{\Phi_{j}\right\}$ constitutes a complete set of statistically orthogonal r.v.'s of the Hilbert space $L_{2}$ with respect to the inner product, i.e., $\left\langle\Phi_{i}, \Phi_{k}\right\rangle=\delta_{i k}\left\langle\Phi_{i}, \Phi_{i}\right\rangle$, where $\langle\cdot\rangle$ denotes the following average

$$
\langle f(\boldsymbol{\xi}), g(\boldsymbol{\xi})\rangle=\int f(\boldsymbol{\xi}) g(\boldsymbol{\xi}) W(\boldsymbol{\xi}) \mathrm{d} \boldsymbol{\xi},
$$

$W(\boldsymbol{\xi})$ is the weighting function corresponding to the Wiener-Askey polynomial chaos basis $\mathcal{B}=\left\{\Phi_{j}\right\}$ and $\delta_{i k}$ is Kronecker delta function. In addition, for $j \geq 1$ these polynomials are centered at the origin, i.e., $\left\langle\Phi_{j}\right\rangle=0, j \geq 1$, and $\Phi_{0}=1$. As a consequence, from (3)-(4) the expectation and variance of r.v. $\chi$ can be computed in terms of coefficients $\chi_{i}$ in the following way

$$
\mathrm{E}_{\mathrm{PC}}^{\mathcal{B}}=\langle\chi(\omega)\rangle=\chi_{0}, \quad \mathrm{D}_{\mathrm{PC}}^{\mathcal{B}}=\operatorname{Var}[\chi(\omega)]=\sum_{i=1}^{\infty}\left(\chi_{i}\right)^{2}\left\langle\left(\Phi_{i}(\boldsymbol{\xi}(\omega))\right)^{2}\right\rangle,
$$

respectively, see [18] for further details.

In the operational practice, the infinite summation (3) needs to be truncated at a finite term, say $P$. The vector $\boldsymbol{\xi}=\left(\xi_{i_{1}}, \xi_{i_{2}}, \ldots\right)$ is also truncated at the number $n$, called the dimension of the chaos, i.e., $\boldsymbol{\xi}=\left(\xi_{1}, \ldots, \xi_{n}\right)$. In our case, this leads to the following expansion of solution s.p. $X(t ; \omega)$ and input r.v.'s $A(\omega), Y_{0}(\omega), Y_{1}(\omega)$

$$
\begin{aligned}
X(t ; \omega) & =\sum_{i=0}^{P} X_{i}(t) \Phi_{i}(\boldsymbol{\xi}(\omega)), & A(\omega) & =\sum_{i=0}^{P} A_{i} \Phi_{i}(\boldsymbol{\xi}(\omega)), \\
Y_{0}(w) & =\sum_{i=0}^{P} Y_{0, i} \Phi_{i}(\boldsymbol{\xi}(\omega)), & Y_{1}(w) & =\sum_{i=0}^{P} Y_{1, i} \Phi_{i}(\boldsymbol{\xi}(\omega)) .
\end{aligned}
$$

In these expansions, the total number of terms is $P+1$. This value is fixed by the relationship $P+1=(n+p) ! /(n ! p !)$, where $n$ is the dimension of the chaos, (i.e., the number of components of vector $\boldsymbol{\xi}$ ) and, $p$, the highest order of the polynomial basis $\mathcal{B}=\left\{\Phi_{i}\right\}$. Since we are going to consider $A, Y_{0}$ and $Y_{1}$ as the input r.v.'s in problem (1), we will take $n=3$, so $\boldsymbol{\xi}(\omega)=\left(\xi_{1}(\omega), \xi_{2}(\omega), \xi_{3}(\omega)\right)$

For the sake of clarity in the presentation, we illustrate the notation above for $p=2$. In this case, the polynomial basis can be chosen as (see for example [18]) 


$$
\begin{array}{lll}
\Phi_{0}=\Gamma_{0}=1, & & \\
\Phi_{1}=\Gamma_{1}\left(\xi_{1}(\omega)\right), & \Phi_{2}=\Gamma_{1}\left(\xi_{2}(\omega)\right), & \Phi_{3}=\Gamma_{1}\left(\xi_{3}(\omega)\right), \\
\Phi_{4}=\Gamma_{2}\left(\xi_{1}(\omega), \xi_{1}(\omega)\right), & \Phi_{5}=\Gamma_{1}\left(\xi_{1}(\omega)\right) \Gamma_{1}\left(\xi_{2}(\omega)\right), & \Phi_{6}=\Gamma_{1}\left(\xi_{1}(\omega)\right) \Gamma_{1}\left(\xi_{3}(\omega)\right) \\
\Phi_{7}=\Gamma_{2}\left(\xi_{2}(\omega), \xi_{2}(\omega)\right), & \Phi_{8}=\Gamma_{1}\left(\xi_{2}(\omega)\right) \Gamma_{1}\left(\xi_{3}(\omega)\right), & \Phi_{9}=\Gamma_{2}\left(\xi_{3}(\omega), \xi_{3}(\omega)\right)
\end{array}
$$

where independence between r.v.'s $\xi_{1}, \xi_{2}$ and $\xi_{3}$ has been considered.

So far, we have used the polynomial basis associated to only one of the polynomials of the Wiener-Askey scheme. If each r.v. $A, Y_{0}$ and $Y_{1}$ is expanded in a different basis, $\Gamma_{i}^{1}, \Gamma_{i}^{2}$ and $\Gamma_{i}^{3}$, respectively, taking into account (2), after truncation we obtain

$$
\begin{gathered}
A(\omega)=\widehat{A}_{0}+\widehat{A}_{1} \Gamma_{1}^{1}\left(\xi_{1}(\omega)\right)+\widehat{A}_{2} \Gamma_{2}^{1}\left(\xi_{1}(\omega), \xi_{1}(\omega)\right) \\
Y_{0}(w)=\widehat{Y}_{0,0}+\widehat{Y}_{0,1} \Gamma_{1}^{2}\left(\xi_{2}(\omega)\right)+\widehat{Y}_{0,2} \Gamma_{2}^{2}\left(\xi_{2}(\omega), \xi_{2}(\omega)\right), \\
Y_{1}(w)=\widehat{Y}_{1,0}+\widehat{Y}_{1,1} \Gamma_{1}^{3}\left(\xi_{3}(\omega)\right)+\widehat{Y}_{1,2} \Gamma_{2}^{3}\left(\xi_{3}(\omega), \xi_{3}(\omega)\right)
\end{gathered}
$$

and the orthogonal basis, in accordance with expression (7), is

$$
\begin{array}{lll}
\Phi_{0}=\Gamma_{0}=1, & & \\
\Phi_{1}=\Gamma_{1}^{1}\left(\xi_{1}(\omega)\right), & \Phi_{2}=\Gamma_{1}^{2}\left(\xi_{2}(\omega)\right), & \Phi_{3}=\Gamma_{1}^{3}\left(\xi_{3}(\omega)\right), \\
\Phi_{4}=\Gamma_{2}^{1}\left(\xi_{1}(\omega), \xi_{1}(\omega)\right), & \Phi_{5}=\Gamma_{1}^{1}\left(\xi_{1}(\omega)\right) \Gamma_{1}^{2}\left(\xi_{2}(\omega)\right), & \Phi_{6}=\Gamma_{1}^{1}\left(\xi_{1}(\omega)\right) \Gamma_{1}^{3}\left(\xi_{3}(\omega)\right), \\
\Phi_{7}=\Gamma_{2}^{2}\left(\xi_{2}(\omega), \xi_{2}(\omega)\right), & \Phi_{8}=\Gamma_{1}^{2}\left(\xi_{2}(\omega)\right) \Gamma_{1}^{3}\left(\xi_{3}(\omega)\right), & \Phi_{9}=\Gamma_{2}^{3}\left(\xi_{3}(\omega), \xi_{3}(\omega)\right) .
\end{array}
$$

Now, we are ready to explain how the polynomial chaos operational methodology works in model (1). Firstly, we impose that the truncated polynomial chaos series given by (6) satisfies the random Airy differential equation (1)

$$
\sum_{i=0}^{P} \ddot{X}_{i}(t) \Phi_{i}(\boldsymbol{\xi}(\omega))+t \sum_{i=0}^{P} \sum_{j=0}^{P} A_{i} X_{j}(t) \Phi_{i}(\boldsymbol{\xi}(\omega)) \Phi_{j}(\boldsymbol{\xi}(\omega))=0 .
$$

A Galerkin projection of previous equation onto each polynomial basis $\mathcal{B}=\left\{\Phi_{i}\right\}$ is then conducted in order to ensure the error is orthogonal to the functional space spanned by the finite-dimensional basis $\mathcal{B}=\left\{\Phi_{i}\right\}$

$$
\begin{gathered}
\sum_{i=0}^{P} \ddot{X}_{i}(t)\left\langle\Phi_{i}(\boldsymbol{\xi}(\omega)), \Phi_{l}(\boldsymbol{\xi}(\omega))\right\rangle \\
+t \sum_{i=0}^{P} \sum_{j=0}^{P} A_{i} X_{j}(t)\left\langle\Phi_{i}(\boldsymbol{\xi}(\omega)) \Phi_{j}\left(\xi_{1}(\omega)\right), \Phi_{l}(\boldsymbol{\xi}(\omega))\right\rangle=0, \quad l=0,1, \ldots, P .
\end{gathered}
$$


Now, taking advantage of orthogonality properties of polynomial basis $\mathcal{B}=\left\{\Phi_{i}\right\}$, one obtains the following coupled second-order system of deterministic differential equations

$$
\begin{aligned}
& \ddot{X}_{l}(t)=-\frac{t}{e_{l}} \sum_{i=0}^{P} \sum_{j=0}^{P} e_{i j l} A_{i} X_{j}(t), \quad l=0,1, \ldots, P, \\
& X_{l}(0)=Y_{0, l}, \quad \dot{X}_{l}(0)=Y_{1, l}
\end{aligned}
$$

where

$$
e_{i j l}=\left\langle\Phi_{i}(\boldsymbol{\xi}(\omega)) \Phi_{j}(\boldsymbol{\xi}(\omega)), \Phi_{l}(\boldsymbol{\xi}(\omega))\right\rangle, \quad i, j, l=0,1, \ldots, P
$$

$$
e_{l}=\left\langle\left(\Phi_{l}(\boldsymbol{\xi}(\omega))\right)^{2}\right\rangle, \quad A_{i}=\frac{\left\langle A, \Phi_{i}(\boldsymbol{\xi}(\omega))\right\rangle}{\left\langle\left(\Phi_{i}(\boldsymbol{\xi}(\omega))\right)^{2}\right\rangle}, \quad i, l=0,1, \ldots, P .
$$

In the significant case where $A$ is a r.v. of the same class of $\xi$, according to expression (4) the coefficients $A_{i}$ can still be computed in the same way that $e_{l}$ and $e_{i j l}$. Whereas if $A$ is not of the same type, the computation of the numerator defining coefficients $A_{i}$ requires the transformation of r.v.'s, $A$ and $\xi$ to the same uniformly distributed r.v. $U$ by using the inverse transformation method [24]. This can be done as follows

$$
\left\langle A, \Phi_{i}(\xi(\omega))\right\rangle=\int_{0}^{1} F_{A}^{-1}(u) \Phi_{i}\left(F_{\xi}^{-1}(u)\right) \mathrm{d} u, \quad i=0,1, \ldots, P,
$$

where $F_{H}^{-1}$ denotes the inverse distribution function of r.v. $H$.

\section{Numerical results}

As we pointed out in Section 1, we are interested in studying how solutions s.p. to r.d.e.'s depend on the statistical distributions of the random model parameters (inputs and initial conditions) as well as the chosen basis when applying gPC. As we said in Section 1, the elucidation of this last question is of paramount importance in the case that random model parameters have different statistical distributions, when the Hermite basis is often chosen to represent the random model parameters and the solution s.p. In fact, keeping in mind the idea of developing both, the random data and the solution s.p., with respect to a single basis, we will show that the consideration of, just, the Hermite basis to perform these developments may not be 
an appropriate choice. Instead, we propose to select the polynomial basis in accordance with the random data that most determine the behavior of the model.

By the reasons exhibited in Section 1, we will consider the Airy random differential equation to conduct this study. Specifically, we will compute the solution s.p. to r.d.e. (1) considering that random model parameters $A, Y_{0}$, $Y_{1}$ have the Gaussian $(\mathrm{N})$ and uniform $(\mathrm{U})$ statistical distributions specified in Table 1. The distributions of $A, Y_{0}, Y_{1}$ have been selected so that each one of them has the same mean and variance in all Cases 1-8. Thus, we can highlight how important is correctly setting the probability distributions of the data. Namely in Table 1, the parameters $\{a, b\},\{c, d\}$ and $\{e, f\}$, associated to the uniform distributions $U(a, b), U(c, d)$ and $U(e, f)$, have been fixed in such a way that their mean and variance match those ones of the Gaussian r.v.'s $A \sim \mathrm{N}(1,1 / 4), Y_{0} \sim \mathrm{N}(1,1)$ and $Y_{1} \sim \mathrm{N}(1 / 3,1 / 25)$, respectively. For instance, in the first case, $a$ and $b$ have been determined so that the uniform distribution $U(a, b)$ has mean 1 and variance $1 / 4$, and analogously, for the parameters $c, d, e$ and $f$. Thus, we have obtained the following values

$$
\begin{array}{lll}
a=\frac{4-\sqrt{3}}{2}, & c=1-\sqrt{3}, & e=\frac{5-3 \sqrt{3}}{15} \\
b=\frac{3+4 \sqrt{3}}{2 \sqrt{3}}, & d=\frac{3+\sqrt{3}}{\sqrt{3}}, & f=\frac{9+5 \sqrt{3}}{15 \sqrt{3}} .
\end{array}
$$

To study whether there is dependence on the chosen basis when the gPC is applied for each of the eight cases collected in Table 1, following the recommendations given by [19], we have taken the Hermite and Legendre polynomials bases, associated to Gaussian and uniform r.v.'s, respectively.

First, we consider that every random model parameter $A, Y_{0}$ and $Y_{1}$ has a Gaussian distribution. This corresponds to Case 1 in Table 1. For simplicity, it has been denoted by NNN. In this scenario, as all r.v.'s are Gaussian, based on [19], we have used the Hermite orthogonal polynomials basis to approximate the average and standard deviation by gPC. Henceforth, this will denoted by Hermite-gPC. In Figure 1, we show the calculated results for different orders, namely 1,2 and 5 , of $\mathrm{gPC}$ as well as the results using the Monte Carlo method with $5 \times 10^{5}$ with the simulations done over the time interval $[0,5]$, where we set our discussion. Notice that in the case of the average, the approximations provided by both approaches match very well even for gPC with order 2 , while a higher order, namely 5 , is needed for a good 


\begin{tabular}{ccccc}
\hline Case & $A$ & $Y_{0}$ & $Y_{1}$ & Notation \\
\hline 1 & $\mathrm{~N}(1,1 / 4)$ & $\mathrm{N}(1,1)$ & $\mathrm{N}(1 / 3,1 / 25)$ & $\mathrm{NNN}$ \\
2 & $\mathrm{~N}(1,1 / 4)$ & $\mathrm{N}(1,1)$ & $\mathrm{U}(e, f)$ & $\mathrm{NNU}$ \\
3 & $\mathrm{~N}(1,1 / 4)$ & $\mathrm{U}(c, d)$ & $\mathrm{N}(1 / 3,1 / 25)$ & $\mathrm{NUN}$ \\
4 & $\mathrm{~N}(1,1 / 4)$ & $\mathrm{U}(c, d)$ & $\mathrm{U}(e, f)$ & $\mathrm{NUU}$ \\
5 & $\mathrm{U}(a, b)$ & $\mathrm{N}(1,1)$ & $\mathrm{N}(1 / 3,1 / 25)$ & $\mathrm{UNN}$ \\
6 & $\mathrm{U}(a, b)$ & $\mathrm{N}(1,1)$ & $\mathrm{U}(e, f)$ & $\mathrm{UNU}$ \\
7 & $\mathrm{U}(a, b)$ & $\mathrm{U}(c, d)$ & $\mathrm{N}(1 / 3,1 / 25)$ & $\mathrm{UUN}$ \\
8 & $\mathrm{U}(a, b)$ & $\mathrm{U}(c, d)$ & $\mathrm{U}(e, f)$ & $\mathrm{UUU}$ \\
\hline
\end{tabular}

Table 1: Specification of Cases 1-8 considered to study whether there is dependence on the chosen basis when gPC is applied. In each case, random model parameters $A, Y_{0}$ and $Y_{1}$ are assumed to be Gaussian $(\mathrm{N})$ and uniform $(\mathrm{U})$.

match for the standard deviation. This comparative study between gPC and Monte Carlo methods, allows us to consider as reliable those Hermite-gPC approximations on the interval $[0,5]$ whose order is greater than 5 .

Then, in order to compare the results obtained in Cases 1-4, hereinafter we need to take a true or reference solution. This true solution has been constructed so that the maximum difference on the interval $[0,5]$ between two approximations of consecutive orders of the standard deviation obtained by Hermite-gPC is less than $10^{-12}$. Specifically, the solution constructed in this way corresponds to that one obtained by applying Hermite-gPC with order 17 .
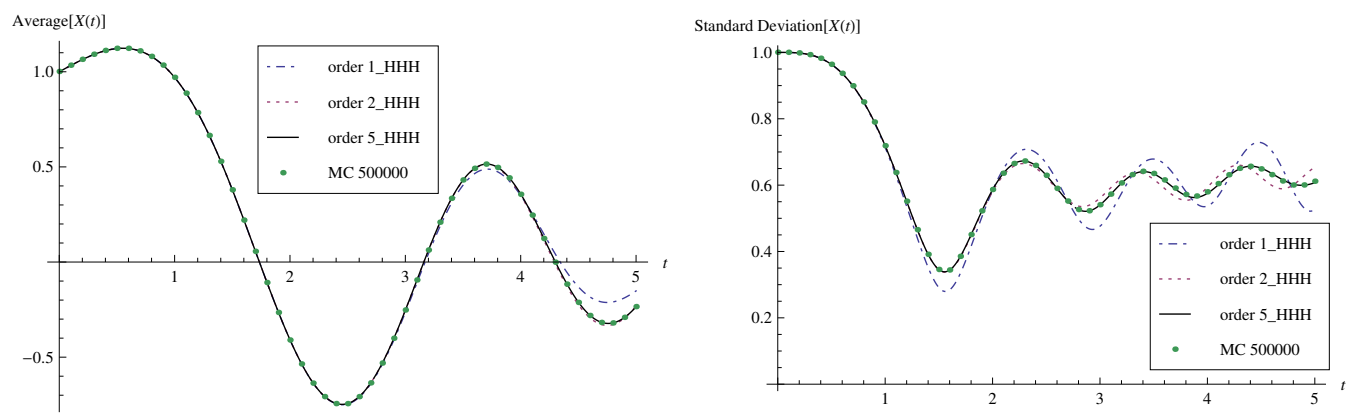

Figure 1: Approximations of the average and the standard deviation to model (1) in Case 1 of Table 1 by using different orders of Hermite-gPC and Monte Carlo with $5 \times 10^{5}$ simulations. 
Approximations for the standard deviation for the Cases 1-4 have been carried out until the difference between two consecutive orders has been less than $5 \times 10^{-3}$, when a numerical stabilization of the results is presented. In Figure 2, we have represented, in semi-logarithmic scale, the relative error of the standard deviation for stabilized Cases 1-4 with respect to the reference solution. Notice that, the plot labels in Figure 2 indicate in the first place the case under study according to the notation introduced in Table 1 and, second, the type of orthogonal polynomial basis used to represent each of the random model parameters that, in this case, it corresponds to the Hermite (H) polynomials. For instance, NUU_HHH notation refers to Case 4 and it indicates that r.v. A, which is assumed to be Gaussian, has been represented by gPC through Hermite polynomials, and r.v.'s $Y_{0}, Y_{1}$, which are assumed to be uniform, have also been represented by Hermite-gPC. The magnitudes of the errors shown in Figure 2 indicate that the statistical distributions of the initial conditions $Y_{0}, Y_{1}$ are not crucial to determine good approximations for the average and standard deviation of the s.p. solution by Hermite-gPC in each of Cases 1-4.

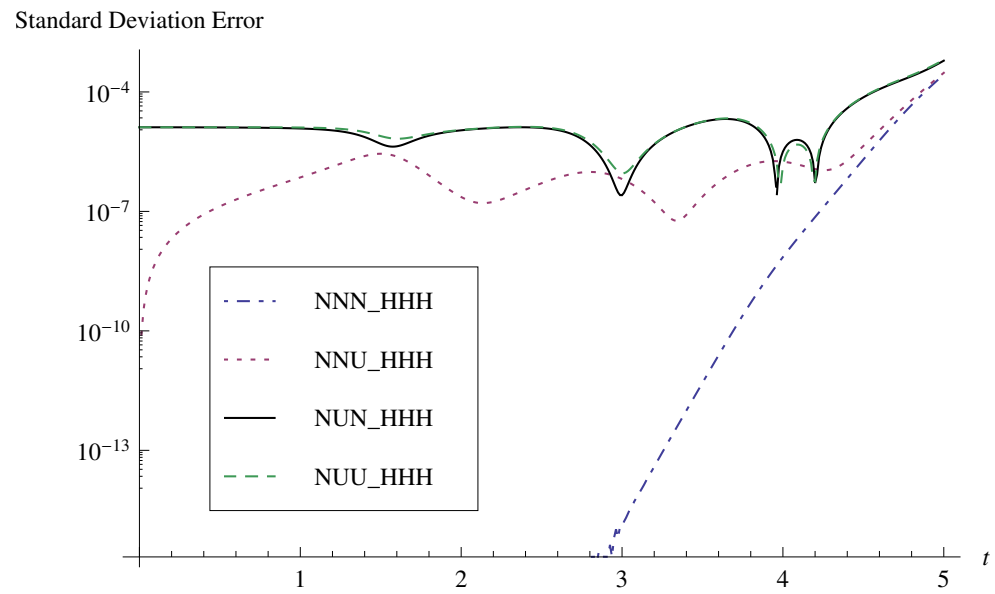

Figure 2: Relative error, in semi-logarithmic scale, of the standard deviation for stabilized Cases 1-4 collected in Table 1 with respect to the so-called reference solution. First part of the plot labels indicates the probability distribution of each one of the random model parameters $A, Y_{0}$ and $Y_{1}$, respectively, according to Cases 1-4, while the second part stands for the orthogonal polynomial basis used to represent them, respectively. In this case, we have just used Hermite $(\mathrm{H})$ polynomial basis. 
In order to analyze the influence of the chosen distribution for the parameter $A$ in the determination of the approximate solution obtained by gPC, in Figure 3 we have plotted the Case 5 for different orders of Hermite-gPC together with the approximation computed by Monte Carlo with $5 \times 10^{5}$ simulations and the reference solution obtained in the Case 1 (NNN_HHH). On the one hand, we observe that although r.v. $A$ has a uniform distribution and it has been represented through the Hermite polynomials, the results provided by Hermite-gPC and Monte Carlo agree. On the other hand, we conclude that the obtained solution differs from that one computed in the Case 1, where the r.v. $A$ is assumed to be Gaussian and the distributions for initial conditions $Y_{0}$ and $Y_{1}$ do not change. The analysis above, allows us to reach the conclusion that the statistical distribution of r.v. $A$ influences in the determination of the solution to model (1).
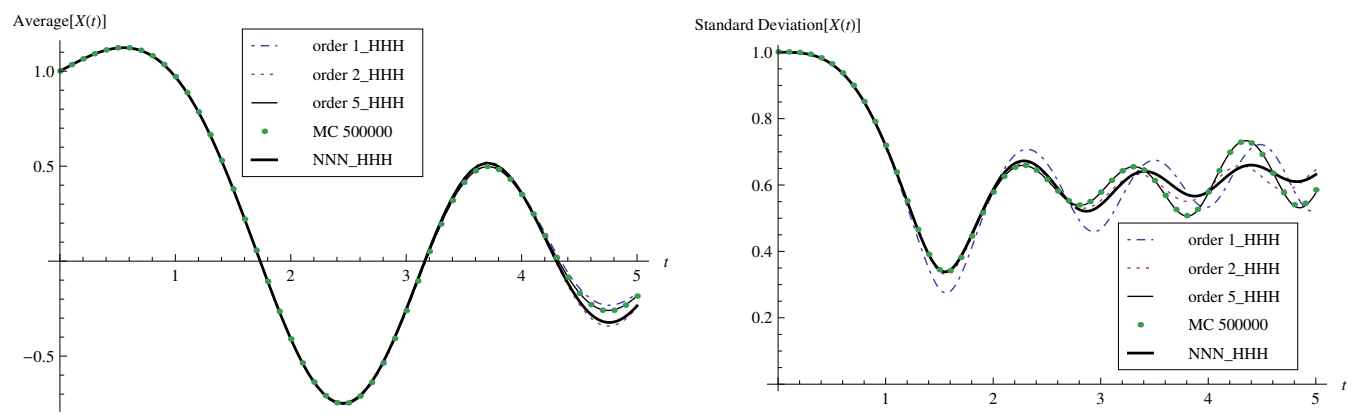

Figure 3: Approximations of the average and standard deviation to model (1) in Case 5 of Table 1 by using different orders of Hermite-gPC, Monte Carlo method with $5 \times 10^{5}$ simulations and the so-called reference solution obtained in the Case 1 (NNN_HHH) by Hermite-gPC.

In Figure 4 we have represented, in semi-logarithmic scale, the relative error of the standard deviation for the stabilized approximations computed in Cases $5-8$ by Hermite-gPC with respect to the so-called reference solution. From this plot, we see again that the statistical distributions of the initial conditions $Y_{0}$ and $Y_{1}$ are not decisive to construct reliable approximations of the solution s.p. to model (1). Comparing the numerical values of the errors represented in Figures 2 and 4, it is clear that those ones corresponding to Figure 4 are greater, so we conclude that the probability distribution of r.v. $A$ has a significant influence on the approximations constructed by HermitegPC. 


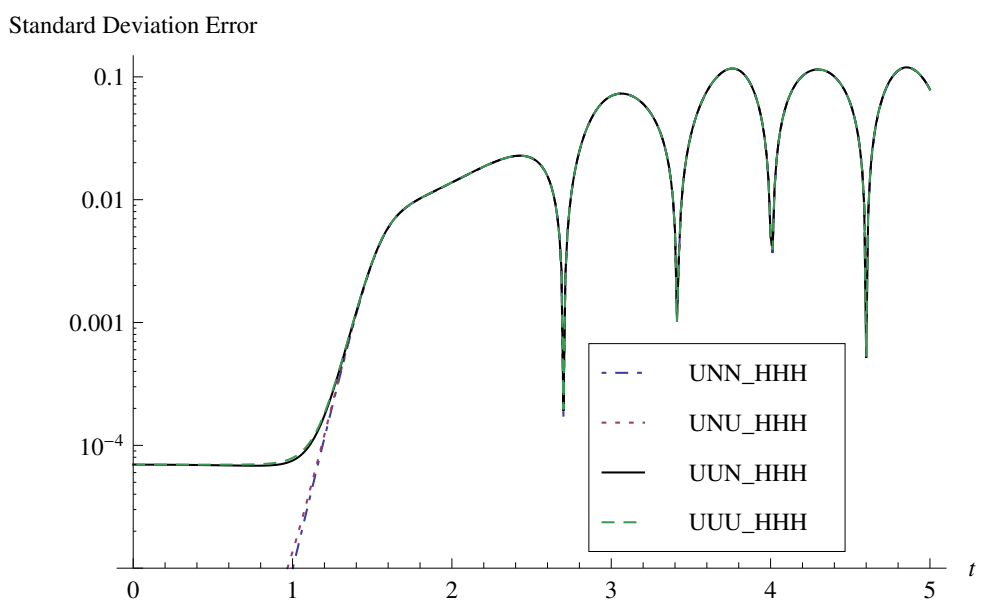

Figure 4: Relative error, in semi-logarithmic scale, of the standard deviation for stabilized Cases 5-8 collected in Table 1 with respect to the so-called reference solution constructed by Hermite-gPC. First part of the plot labels indicates the probability distribution of each one of the random model parameters $A, Y_{0}$ and $Y_{1}$, respectively, according to Cases 5-8, while the second part stands for the orthogonal polynomial basis used to represent them, respectively. In this case, we have just used Hermite $(\mathrm{H})$ polynomial basis. 


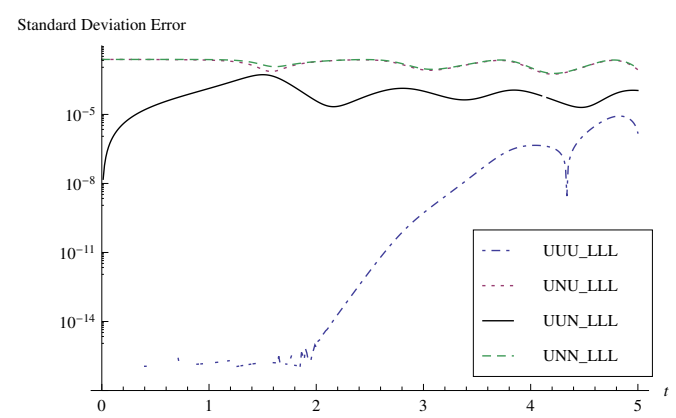

To confirm that conclusions drawn by Hermite-gPC do not depend on the chosen orthogonal polynomial basis, in Figure 5 we show an analogous study to that one we have performed in Figures 2 and 4 for Cases 1-8, but now using Legendre-gPC. Following the same criterion of numerical stabilization we previously used for Hermite-gPC, in this case the approximations of the average and standard deviation have been computed by Legendre-gPC with orders 6 and 9, respectively. As reference solution, now we have taken that one associated to Case 8 in Table 1 constructed, in accordance with [19], by Legendre-gPC with order 15. Again, as we made in the Hermite-gPC analysis, this true solution has been constructed so that the maximum difference on the interval $[0,5]$ between two approximations of consecutive orders of the standard deviation obtained by Legendre-gPC is less than $10^{-12}$. From plots shown in Figure 5, we observe that the approximations provided by Legendre-gPC do not depend on the probability distributions of the initial conditions $Y_{0}$ and $Y_{1}$. Whereas comparing the magnitudes of the errors represented in each plot, we conclude that the constructed approximations depend on the probability distribution of the input r.v. $A$.

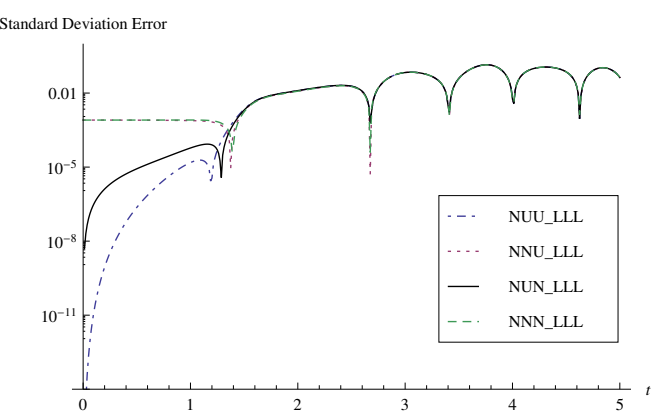

Figure 5: Relative errors, in semi-logarithmic scale, of the standard deviation for stabilized Cases 1-4 (right) and Cases 5-8 (left) shown in Table 1 with respect to the so-called reference solution constructed by Legendre-gPC. First part of the plot labels indicates the probability distribution of each one of the random model parameters $A, Y_{0}$ and $Y_{1}$, respectively, according to Cases $1-8$, while the second part stands for the orthogonal polynomial basis used to represent them, respectively. In this case, we have just used Legendre (L) polynomial basis.

So far we have discussed how to influence the probability distributions of r.v.'s $A, Y_{0}$ and $Y_{1}$ in the determination of the approximation of the solution s.p. to model (1) by gPC method. Our analysis allows us to conclude that 
the choice of the right probability distribution to input r.v. $A$ is particularly crucial, whereas the selection of the correct probability distributions of the initial conditions is not as critical.

This conclusion can be strengthened from the so-called gPC-based Sobol' indices [25]. The Sobol' indices are known to be good descriptors of the sensitivity of the model to its random input parameters (in our case they correspond to $A, Y_{0}$ and $Y_{1}$ ), through r.v.'s of the chosen basis (in our case they are denoted by $\xi_{1}, \xi_{2}$ and $\xi_{3}$, respectively). These descriptors evaluate the part of the total variability of the solution s.p. that is explained by each random model parameter through its contribution by gPC expansion. Although the part of the total variability that determines each random model parameter is not enough to describe completely the statistical distribution of the response, it gives a feeling of the role that each parameter plays in determining the solution s.p. Let us represent each multivariate polynomial of the chosen basis $\mathcal{B}$ (that in our previous development, it corresponds to Hermite or Legendre bases), by means of a $n$-tuple $\boldsymbol{\alpha}=\left(\alpha_{1}, \ldots, \alpha_{n}\right)$ in accordance with gPC construction (see [25, Appendix A] for further details). In this context, polynomials $\Phi_{i}$ and $\Phi_{\boldsymbol{\alpha}}$ are used indifferently according to:

$$
\Phi_{i} \equiv \Phi_{\boldsymbol{\alpha}}: \quad \Phi_{i}(\boldsymbol{\xi})=\prod_{j=1}^{n} P_{\alpha_{j}}\left(\xi_{j}\right),
$$

where $P_{k}(\xi)$ denotes the $k$-th (Hermite or Legendre) univariate orthogonal polynomial belonging to basis $\mathcal{B}$. Then, defining $\mathcal{I}_{i_{1}, \ldots, i_{s}}$ the set of $\boldsymbol{\alpha}$-tuples such that only the indices $\left(i_{1}, \ldots, i_{s}\right)$ are nonzero, the gPC-based Sobol's indices with respect to basis $\mathcal{B}$ are defined as

$$
S_{i_{1}, \ldots, i_{s}}^{\mathcal{B}}=\frac{\sum_{\boldsymbol{\alpha} \in \mathcal{I}_{i_{1}, \ldots, i_{s}}}\left(\chi_{\boldsymbol{\alpha}}\right)^{2}\left\langle\left(\Phi_{\boldsymbol{\alpha}}\right)^{2}\right\rangle}{\mathrm{D}_{\mathrm{PC}}^{\mathcal{B}}}
$$

where $\mathrm{D}_{\mathrm{PC}}^{\mathcal{B}}$ is given in (5). Notice that in the numerator of (8) the gPC expansion coefficients are simply gathered according to the dependency of each basis polynomial, square-summed and normalized. It is important to stress that the sum defining $S_{i_{1}, \ldots, i_{s}}^{\mathcal{B}}$ indicates implicitly the dependence of each multidimensional (Hermite $(\mathrm{H})$, Legendre $(\mathrm{L}))$ orthogonal polynomial to each subset of random input parameters through their identification with the r.v.'s of the chosen basis. In particular, note that $\mathcal{I}_{i}$ corresponds to the orthogonal polynomials depending on a single r.v. $\xi_{i}$ of the chosen basis. 
Therefore, in this case the value of the Sobol' index $S_{i}^{\mathcal{B}}$ rates the part of the total variability which is explained by r.v. $\xi_{i}$ (or equivalently, by the random model parameter that it represents). Considering the identification $\xi_{1} \rightarrow A$, $\xi_{2} \rightarrow Y_{0}$ and $\xi_{3} \rightarrow Y_{1}$ for each of the Cases 1-8 shown in Table 1, we can compute the gPC-based Sobol's indices $S_{A}^{\mathcal{B}}, S_{Y_{0}}^{\mathcal{B}}$ and $S_{Y_{1}}^{\mathcal{B}}$ to the approximate solution s.p. expanded in both bases, $\mathcal{B}=\{\mathrm{H}, \mathrm{L}\}$. Notice that in this context such indices depend implicitly on time $t$. In Table 2 we collect Sobol's indices at the endpoint $t=5$. Notice that the numerical values corresponding to $S_{A}^{\mathcal{B}}$ are greater than those ones associated to $S_{Y_{i}}^{\mathcal{B}}, i=0,1$. This indicates that random input parameter $A$ contributes more to explain the central second moment of the approximate solution s.p. than initial conditions $Y_{0}$ and $Y_{1}$. Thus, this conclusion drawn by Sobol' indices agrees with that one we have obtained previously.

\begin{tabular}{ccccccc}
\hline Case & $S_{A}^{\mathrm{H}}$ & $S_{Y_{0}}^{\mathrm{H}}$ & $S_{Y_{1}}^{\mathrm{H}}$ & $S_{A}^{\mathrm{L}}$ & $S_{Y_{0}}^{\mathrm{L}}$ & $S_{Y_{1}}^{\mathrm{L}}$ \\
\hline 1 (NNN) & 0.54530 & 0.05836 & 0.00618 & 0.53151 & 0.06122 & 0.00663 \\
2 (NNU) & 0.54534 & 0.05837 & 0.00618 & 0.53150 & 0.06122 & 0.00666 \\
3 (NUN) & 0.54567 & 0.05840 & 0.00618 & 0.53129 & 0.06140 & 0.00664 \\
4 (NUU) & 0.54571 & 0.05840 & 0.00618 & 0.53127 & 0.06140 & 0.00666 \\
5 (UNN) & 0.52310 & 0.04425 & 0.00388 & 0.52381 & 0.04371 & 0.00382 \\
6 (UNU) & 0.52315 & 0.04425 & 0.00388 & 0.52369 & 0.04370 & 0.00385 \\
7 (UUN) & 0.52257 & 0.04420 & 0.00388 & 0.52289 & 0.04391 & 0.00382 \\
8 (UUU) & 0.52264 & 0.04420 & 0.00387 & 0.52278 & 0.04390 & 0.00385 \\
\hline
\end{tabular}

Table 2: Numerical values of gPC-based Sobol's indices at $t=5$ with respect to bases Hermite (H) and Legendre (L) for Cases 1-8 of Table 1.

In the following, we analyze the role that the chosen polynomial basis plays in the determination of the solution. To perform this study, first we have represented in Figure 6 the standard deviation corresponding to Case 1 in Table 1 with respect to both bases, Hermite and Legendre. Computations have been carried out on the interval $[0,5]$ by using Hermite-gPC and Legendre-gPC of order 10, as well as, by Monte Carlo with $5 \times 10^{5}$ simulations. We observe that both approximations generated by gPC agree with Monte Carlo results except at the end of the interval where discrepancies with respect to Legendre-gPC values are presented. This reveals the great importance of the chosen orthogonal polynomial basis in order to get better approximations by gPC. 


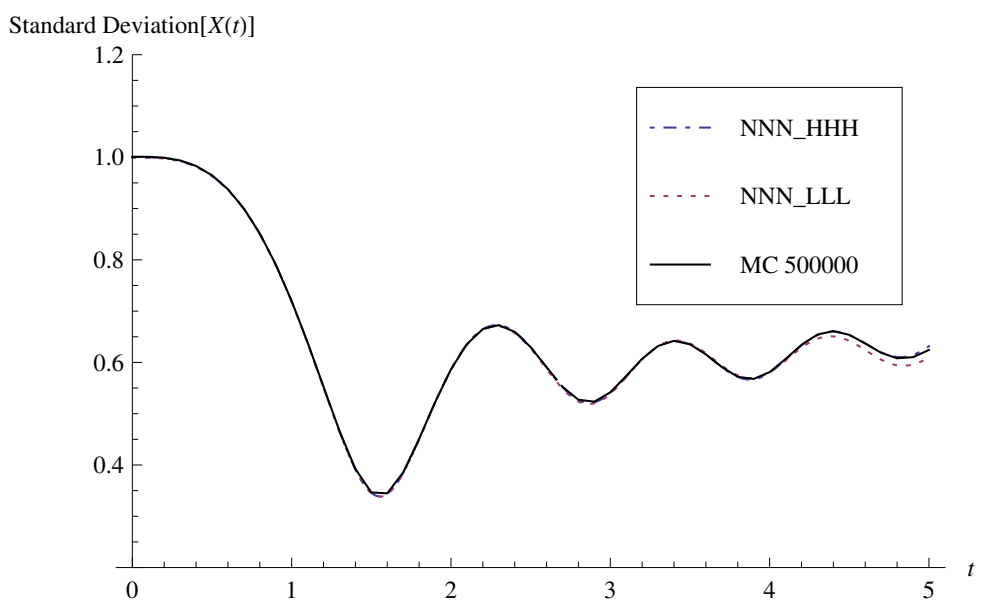

Figure 6: Comparison of the approximations of the stabilized standard deviation in Case 1 of Table 1 by Hermite-gPC (NNN_HHH) and Legendre-gPC (NNN_LLL) with respect to Monte Carlo values using $5 \times 10^{5}$ simulations.

This motivates the subsequent analysis of the results obtained when approximations are computed by adapting completely gPC method to the problem under study. Hereinafter, we refer to as tailor-made-gPC this approach. Tailor-made-gPC consists of representing each independent random model parameter in terms of the orthogonal polynomial basis, say $\mathcal{B}_{i}$, in accordance with conclusions given in [19]. Then, by taking advantage of independence, the solution s.p. is expressed in terms of the basis constructed as the product of bases $\mathcal{B}_{i}$. Following this approach, firstly, in Figure 7 we have plotted both, the average and standard deviation for the Case 5 in Table 1 (UNN) with respect to the Legendre basis for the input r.v. $A$ and the Hermite basis for the initial conditions r.v.'s $Y_{0}$ and $Y_{1}$. Notice that computations have been carried out by using this tailor-made-gPC method for different orders. We realize that the approximation of the standard deviation of order 14 computed by gPC matches the approximation provided by Monte Carlo with $5 \times 10^{5}$ simulations but over a longer interval, namely [0,15], than that one we considered in the previous analysis, $[0,5]$. Following an analogous development as we made previously, secondly, we have determined a reference solution for the case under study. This so-called reference solution has been constructed so that the maximum difference on the interval $[0,15]$ between two approximations of consecutive orders of the standard deviation obtained 
by the so-called tailor-made-gPC is less than $10^{-6}$. Specifically, the solution constructed in this way corresponds to that one obtained by applying gPC with order 20. In Figure 8 we have plotted, in semi-logarithmic scale, the relative error of the standard deviation constructed by tailor-made-gPC for different orders, namely, 8, 11, 14 and 17, with respect to the reference solution.
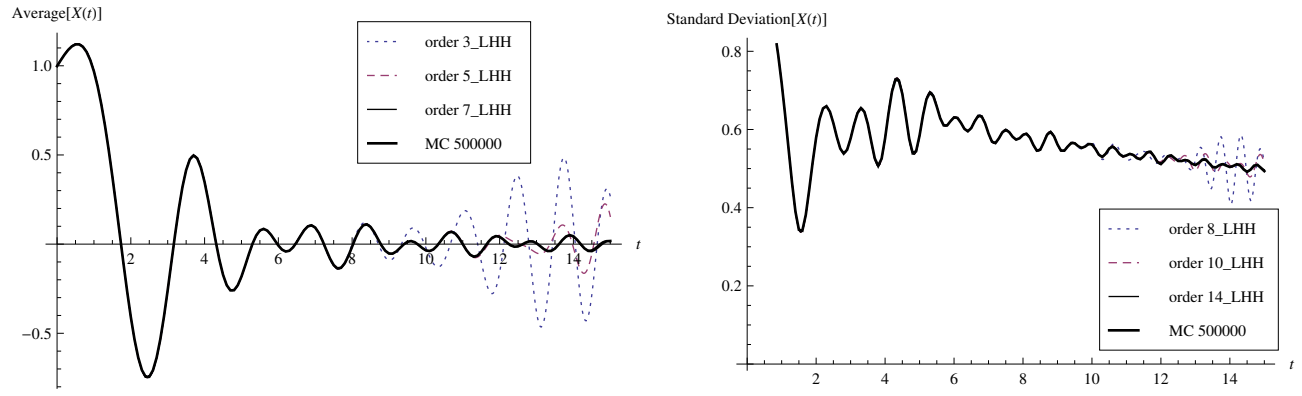

Figure 7: Approximations of the average and standard deviation computed for different orders by gPC for the Case 5 in Table 1 (UNN) using the Legendre basis for the input r.v. $A$ and the Hermite basis for the initial conditions r.v.'s $Y_{0}$ and $Y_{1}$. These approximations are compared with respect to those ones computed by Monte Carlo using $5 \times 10^{5}$ simulations.

In Figure 9 we have represented, in semi-logarithmic scale, the relative error of the standard deviation by Legendre-gPC (left) and Hermite-gPC (right) for different orders with respect to the reference solution constructed by the so-called tailor-made-gPC for the Case 5 in Table 1 (UNN). We notice that the maximum order used to construct the approximations by HermitegPC has been 8 since for higher orders the approximations deteriorate.

By comparing the numerical values of the errors represented in Figure 9, we observe that the approximations provided by Legendre-gPC are better than those ones obtained by Hermite-gPC. Finally, a new comparison between errors shown in Figure 8 and Figure 9 (left) reveals that the approximations can still be improved by using the so-called tailor-made-gPC.

\section{Conclusions and suggestions}

Over the last few decades, random differential equations have demonstrated to be a powerful tool to model problems appearing in applied areas such as physics, medicine, epidemiology, etc. This modelling requires setting 


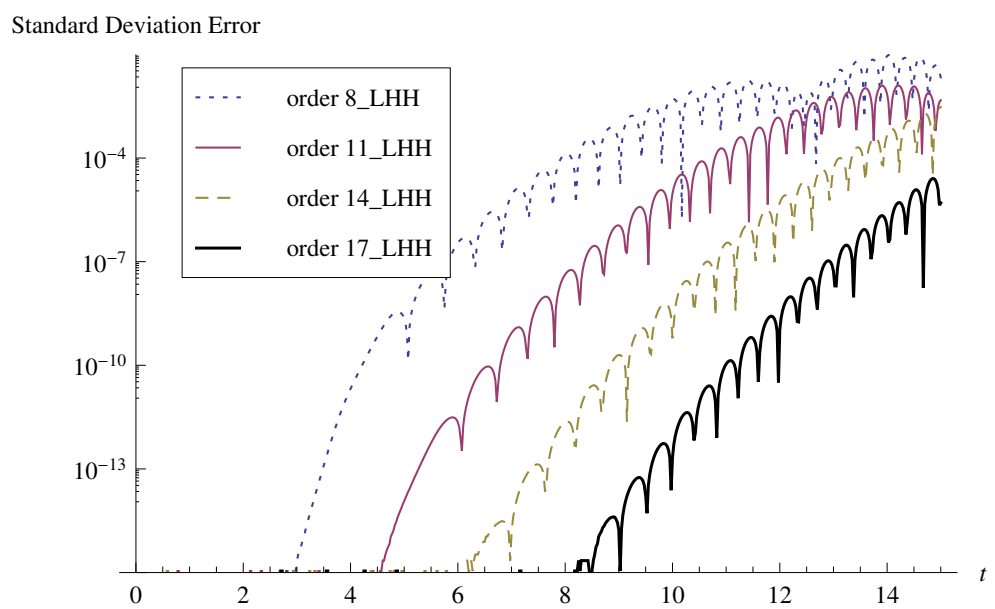

Figure 8: Relative error, in semi-logarithmic scale, of the standard deviation constructed by tailor-made-gPC for different orders, namely, 8, 11, 14 and 17 , with respect to the so-called reference solution constructed by tailor-made-gPC for the Case 5 in Table 1 $(\mathrm{UNN})$.
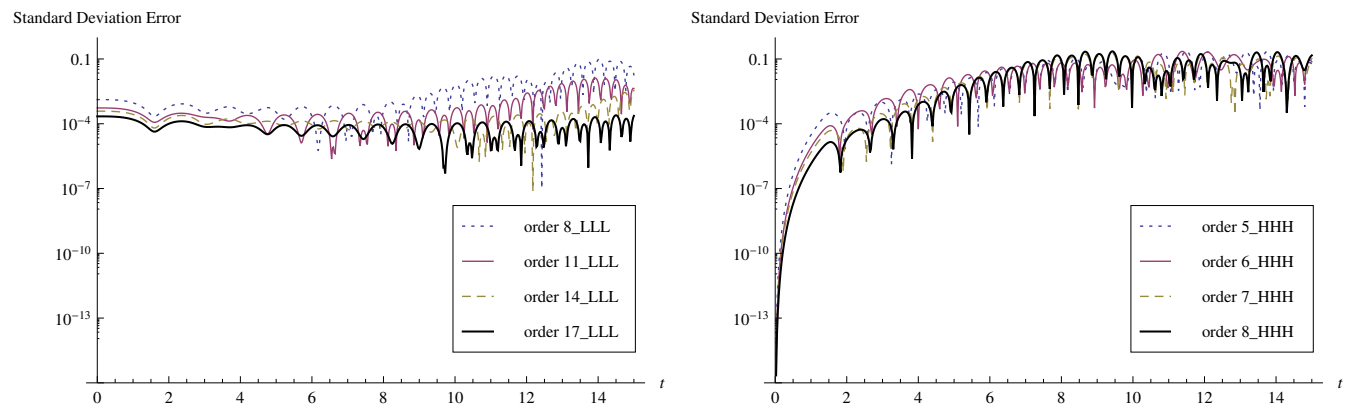

Figure 9: Relative errors, in semi-logarithmic scale, of the standard deviation constructed by Legendre-gPC (left) and by Hermite-gPC (right) for different orders with respect to the reference solution obtained by the so-called tailor-made-gPC for the Case 5 in Table 1 (UNN). 
the statistical distributions of the random model parameters. In practice, the right choice of these distributions can be very difficult due not only to the inherent complexity of the phenomenon under study, but also by the measurement errors that usually contain the samples required to construct such distributions. As a consequence, only approximate distributions for the random model parameters are available. Therefore, an analysis about how this affects the computation of the solution stochastic process to random differential equation is demanded. In this paper we have performed this study for generalized Polynomial Chaos ( $\mathrm{gPC}$ ) which constitutes one of the most powerful methods to deal with the solution of random differential equations. The obtained results have been compared with respect to those ones provided by Monte Carlo technique. To conduct this study we have chosen the random Airy differential equation (1) because of it has highly oscillatory solutions, what allows us to highlight differences when changing the statistical distribution of random inputs (coefficient $A$ and initial conditions $Y_{0}$ and $Y_{1}$ ). Our study shows that setting correctly the distributions of the random model parameters plays an important role in dealing with the solution of random differential equations by gPC. In the specific case of equation (1), we have shown that it is most crucial to fix correctly the statistical distribution associated to the input r.v. $A$ rather than those ones associated to $Y_{0}$ and $Y_{1}$. This conclusion has also been supported by gPC-based Sobol' indices.

The application of gPC entails implicitly the trial choice of an orthogonal polynomial basis. Then, once the statistical distributions of the random model parameters have been set, another significant issue is to analyze whether the chosen basis influences the determination of the solution. In this paper, we have answered this question by considering both, the Hermite and Legendre orthogonal polynomial bases.

In dealing with random models containing just one single random input, the choice of the orthogonal polynomial basis to represent the inputs and the solution can be made according to recommendations given in [19]. For more random inputs, the Hermite polynomials are usually chosen to represent every random parameter and also the solution. In this case, our study shows that this single trial basis should be determined in two steps. First, analyzing the random model parameter that most influences the determination of the solution. Second, choosing the orthogonal polynomial basis associated to this random model parameter in accordance with [19]. However, we conclude our study showing that previous results can be further improved by constructing the solution of the random model through a tailor-made-gPC method based 
on representing every random model parameter in the adequate basis in accordance with [19] and, then constructing the solution by the corresponding orthogonal polynomial bases.

\section{Acknowledgements}

This work has been partially supported by the Spanish M.C.Y.T. and FEDER grants MTM2009-08587, DPI2010-20891-C02-01 as well as the Universitat Politècnica de València grants PAID-00-11 (ref. 2751) and PAID-0611 (ref. 2070).

[1] M. A. El-Tawil, N. A. Al-Mulla, Using homotopy WHEP technique for solving a stochastic nonlinear diffusion equation, Mathematical and Computer Modelling 51 (9-10) (2010) 1277-1284.

[2] M. A. El-Tawil, The average solution of a stochastic nonlinear Schrödinger equation under stochastic complex non-homogeneity and complex initial conditions, in: Trans. Comput. Sci. III, LNCS, Vol. 5300 of Lecture Notes in Computer Science, Springer Verlag, 2009, pp. 143170 .

[3] B. M. Chen-Charpentier, D. Stanescu, Epidemic models with random coefficients, Mathematical and Computer Modelling 52 (7-8) (2009) 1004-1010.

[4] G. Calbo, J. C. Cortés, L. Jódar, Random matrix difference models arising in long-term medical drug strategies, Applied Mathematics and Computation 217 (5) (2010) 2149-2161.

[5] C. Braumann, Variable effort fishing models in random enviroments, Mathematical Biosciences 156 (1-2) (1999) 1-19.

[6] A. J. Arenas, G. González-Parra, J. A. Moraño, Stochastic modelling of the transmission of respiratory synctytial virus (RSV) in the region of Valencia (Spain), ByoSystems 206 (3) (2009) 206-212.

[7] I. I. Gihman, A. V. Skorohod, Stochastic Differential Equations, Springer-Verlag, Berlin, 1972.

[8] T. T. Soong, Random Differential Equations in Science and Engineering, Academic Press, New York, 1973. 
[9] N. Bellomo, R. Riganti, Nonlinear Stochastic Systems in Physics and Mechanics, World Scientific, Singapore, 1987.

[10] L. Arnold, Stochastic Differential Equations: Theory and Applications, John Wiley \& Sons, New York, 1974.

[11] P. Kloeden, E. Platen, Numerical Solution of Stochastic Differential Equations, Springer, Berlin, 1999.

[12] A. Jahedi, G. Ahmadi, Application of Wiener-Hermite expansion to non stationary random vibrations of a Duffing Oscillator, Journal of Applied Mechanics 50 (1-3) (1983) 436-442.

[13] A. H. Nayfeh, Problems in Perturbation, John Wiley \& Sons, New York, 1985 .

[14] P.-T. D. Spanos, W. D. Iwan, The existence and uniqueness of solution generated by equivalent linearization, International Journal of NonLinear Mechanics 13 (2) (1978) 71-78.

[15] G. Adomian, Solving Frontier Problems of Physics: The Decomposition Method, Kluwer, Boston, 1994.

[16] J. C. Cortés, L. Jódar, L. Villafuerte, Numerical solution of random differential initial value problems: Multistep methods, Mathematical Methods in the Applied Sciences 34 (1) (2011) 63-75.

[17] G. Calbo, J. C. Cortés, L. Jódar, Mean square power series solution of random linear differential equations, Computers and Mathematics with Applications 59 (1) (2010) 559-572.

[18] R. Ghanem, P. D. Spanos, Stochastic Finite Elements: A Spectral Approach, Dover Publications, Mineola, NJ, 1991.

[19] D. Xiu, G. Karniadakis, The Wiener-Askey polynomial chaos for stochastic differential equations, SIAM Journal on Scientific Computing 24 (2002) 619-644.

[20] B. J. Debusschere, H. N. Najm, P. P. Pébay, O. M. Knio, R. G. Ghanem, O. P. L. Maitre, Numerical challenges in the use of polynomial chaos representations for stochastic processes, Numerical Challenges in the 
$464 \quad$ Use of Polynomial Chaos Representations for Stochastic Processes 26 (2) $465 \quad$ (2004) 698-719.

[21] M. Loève, Probability Theory, Van Nostrand, Princeton, New Jersey, 1963.

[22] N. Wiener, The homogeneus chaos, American Journal of Mathematics 60 (1938) 897-936.

[23] R. H. Cameron, W. T. Martin, The orthogonal development of nonlinear functionals in series of Fourier-Hermite functionals, Annals of Mathematics 48 (1947) 385-392.

[24] S. M. Ross, A Course in Simulation, Macmillan, New York, 1991.

[25] B. Sudret, Global sensitivity analysis using polynomial chaos expansions, Reliability Engineering and System Safety 93 (2008) 964-979. 